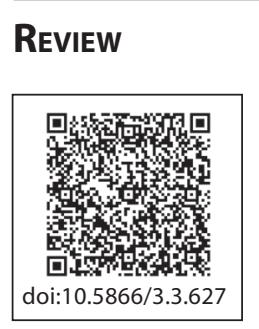

\title{
Full Mouth Rehabilitation with Group Function Occlusal scheme in a patient with severe Dental Fluorosis
}

\author{
Sudhir N' ${ }^{1}$, Hari Parkash ${ }^{2}$
}

Professor ${ }^{1}$

Department of Prosthodontics and Implantology, Kamineni Institute of Dental Sciences, Narketpally, Nalgonda Dist., Andhra Pradesh.

Professor and Director ${ }^{2}$,

I.T.S. Centre for Dental Studies and Research, Muradnagar, Ghaziabad. Uttar Pradesh.

\section{Article Info}

Received: April 12, 2011

Review Completed: May, 16, 2011

Accepted: June, 17, 2011

Available Online: October, 2011

(c) NAD, 2011 - All rights reserved

\begin{abstract}
:
Full mouth rehabilitation continues to be the biggest challenge to any clinician in Restorative dentistry. It requires efficient diagnosis and elaborate treatment planning to develop ordered occlusal contacts and harmonious articulation in order to optimize stomathognathic function, health and esthetics which then translates to patient's comfort and satisfaction. Thorough knowledge of the various concepts of articulation is therefore integral to any full mouth rehabilitation that is taken up to address the patient's problem related to restoration of multiple teeth that are either decayed, worn, broken, discolored, missing or suffer developmental deficits.
\end{abstract}

This case report describes the Full mouth rehabilitation of a 22 year old girl who reported with multiple decayed and discolored fluorosed teeth with proclined anteriors. The case was treated with individual Porcelain fused to metal crowns and a bridge with Group function occlusal scheme using Simultaneous full arch technique which is although exacting, exhaustive and demanding provides for a structured stable and predictable articulation.

Several techniques of Full mouth rehabilitations are available and a clinician should ascribe to one after a comprehensive diagnosis of the patient's clinical condition and prospective consideration of his/her oral health, function, comfort and esthetic requirements.

Key words: Full mouth rehabilitation, Group function, Fluorosis

\section{INTRODUCTION:}

With nearly $14 \%$ of total fluoride available in the Earth's crust present in India, Dental Fluorosis is endemic and is still a serious problem in nearly 15 states of our country. The highest incidence is seen in Andhra Pradesh, Haryana, Karnataka, Punjab, Rajasthan and Tamilnadu. ${ }^{1}$ Fluoride in water is akin to a double edged sword, while it is required in concentrations of $0.5 \mathrm{mg} / \mathrm{L}$ of ground water in tropical countries where the drinking water consumption is more for calcification of the bones and teeth, larger concentrations than this can lead to debilitating dental manifestations that range from mild, moderate to severe. Severe dental fluorosis causes

Email for correspondence:

dr_sudheer_aiims@yahoo.com pitting/mottling of the enamel with increased predisposition to decay, fracture, wear, premature loss leading to degeneration of occlusal stability and hence the masticatory mechanism. It compromises not only the oral health of the patient but also their psychological bearing due to the mutilation and discoloration of affected teeth and impaired function. In many a cases of such severe dental fluorosis Full mouth rehabilitation is not only curative but also preventive and therefore essential.

Of the various treatment procedures in Prosthodontics, the most demanding is the Full mouth Rehabilitation for, it is the culmination of all valid and legible theory and its practical application to provide harmonious occlusal contacts and articulation and synchronize the very delicately 
balanced Stomatognathic system. Full mouth rehabilitations are taken up for various clinical conditions that require extensive prosthetic restorations involving nearly the entire compliment of teeth such as advanced attrition or abrasion and caries causing mutilation of teeth; periodontal involvement with drifted, elongated, extruded teeth with loss of contacts; Temporo mandibular disorders, discolored or malformed teeth secondary to developmental disorders including severe dental fluorosis. $^{2}$ Conformative means of occlusal rehabilitation at the intercuspal position or ICP has to be weighed against the more radical Reorganization of occlusion at the Centric relation contact position or $C R C P$, as it requires exhaustive planning and structured treatment procedures. However, in most cases requiring Full mouth rehabilitations, reorganizing the occlusion is the only means by which we can predict a self-sustained, selfmaintaining masticatory mechanism ensuring health, harmony and comfort.

\section{CASE REPORT:}

A 22 year old girl moderately built with good general health and no systemic ailments reported to the Department of Prosthodontics and Implantology, All India Institute of Medical Sciences, New Delhi. She complained of discolored and forwardly placed anterior teeth and multiple decayed teeth with "chipping off" in the posteriors. The patient gave a history of residence since her birth at a village in Haryana which had high fluoride levels in the ground water and informed of the same problem with her younger siblings. On clinical and radiographic examination it was seen that she suffered from generalized dental fluorosis with severe mottling of the enamel and dark brown discoloration (Deans Fluorosis Index: Severe). Multisurface caries with occlusal wear in all the posterior teeth, missing 24 which was extracted 1 year back due to caries, marked overjet, attrited incisal facets on 11 , $12,21,22,31,32,41,42$, and lower incisal imbrication. Patient was given various treatment options and she consented for a Full mouth rehabilitation. Composite restorations (Solare P, GC corp, Japan) were made in all the carious teeth and RCT was done in 11,12,21,22. Diagnostic impressions were made with irreversible hydrocolloid (Neocolloid, Zhermack, Italy) and the maxillary cast oriented to the articulator axis with a facebow record (Quick mount facebow, Whip mix, U.S.A.). A leaf gauge was used to deprogramme the mandible and was maneuvered into centric closure and a thin trapezoidal Aluwax sheet was used to record the same without any tooth contact (perforation) and the relation used to transfer the mandibular cast to a semi adjustable articulator (Whip mix \#8500, U.S.A.). Protrusive occlusal registration was made with fast setting polyvinylsiloxane bite registration material (Blu Mousse, Parkell, U.S.A.) and the articulator adjusted. Diagnostic occlusal assessment was done and any interference during centric closure noted and removed in the patient's mouth. A permissive occlusal splint fabricated in clear auto-polymerizing resin on the articulated casts was then given both as a definitive deprogrammer and also to assess the possibility of increasing the vertical dimension by $2 \mathrm{~mm}$ if and when required, given her occlusal wear. A diagnostic wax up was made on the articulated models and two Putty Silicone indices (Aquasil, Dentsply, U.S.A.) were made on the Diagnostic wax up models, one complete for fabrication of the temporaries and the other sectioned to aid as a guide during tooth preparation. When after using the splint for 6 weeks, the patient reported of no discomfort, a Reorganized approach to the full mouth rehabilitation with individual porcelain fused to metal crowns and a PFM bridge in relation to 23,24 25 and Group function occlusal scheme through a simultaneous full arch technique was planned. The maxillary and mandibular teeth were prepared simultaneously and impressions made with poly vinyl silicones (Aquasil Putty and Aquasil LV, Dentsply, U.S.A.). Complete arch dies were prepared and the casts mounted using renewed facebow, centric and the protrusive interocclusal records. Temporization (Tooth Shade Acrylic, Duralay, U.S.A.) was done indirectly using the Putty Silicone index from the diagnostic wax up. Metal coping trial was made to confirm fit and marginal integrity. Ceramic (IPS Classic, Ivoclar Vivadent )was fired onto the copings and was followed by the bisque try-in. Final occlusal adjustments to the modified incisal guidance during protrusion were made and Unilateral group function on the working sides was adjusted and confirmed. The crowns and the bridge were finally stained and 
glazed and cemented with a glass ionomer (GC Fuji I) luting cement. Patient was then put on a strict oral hygiene regimen and recall schedule.

\section{DISCUSSION:}

Fixed Prosthodontic procedures fall in one of two categories, Conformative or Re-organized. Conformative occlusal approach is generally followed when relatively small amount of restorative treatment is required and is designed around the patients existing Inter Cuspal Position, ICP (or Maximum Intercuspation, MI) which may or may not coincide with the Centric Relation. It is limited to localized occlusal adjustments involving the tooth to be prepared or its immediate vicinity such as removal of deflecting contact, shortening of the opposing cusp, elimination of non-working side interferences. Reorganized approach is needed when the present ICP is unacceptable and needs to be changed or when extensive restorations are required to optimize the patient's occlusion. Herein, the ICP is developed at the Centric Relation position of the Mandible or the ICP is made coincident with the CRCP (Centric Relation Contact Position). This provides an even and stable occlusion and ensures there are no pathogenic deflective contacts. Therefore, most of all full mouth rehabilitations tread the Reorganized approach as it restores the structural and functional integrity of the dental arches that are compromised extensively by decayed, missing, broken, worn, discolored teeth or teeth suffering from developmental defects or faulty fixed prosthodontic work. Once the decision of full mouth rehabilitation is arrived at, two questions need to be answered, what sequence of tooth preparation and restoration is to be followed and what occlusal scheme to give. Choices range from Simultaneous full arch or Segmental/ Quadrant or Sequential simultaneous techniques and Group function or Canine guided occlusal schemes. Each comes with its own merits and demerits. The choice is balanced between what is best for the patient given the clinical condition and patient's expectation and the operators experience and understanding. Irrespective of the choice, the treatment protocol should essentially include preliminary procedures directed towards eliminating pain, infection, carious lesions and teeth with poor prognosis by an interdisciplinary team of specialists followed by a full analysis of occlusion as it exists.
Analysis of the occlusion requires the transfer of the maxillary and mandibular diagnostic casts to a capable articulator which provides for a more convenient and relatively definite means of assessment. This requires a Facebow record to orient the maxillary cast to the opening and closing axis of the articulator and a set of interocclusal records, one the centric relation record and the other the protrusive and laterotrusive records which are used to transfer the mandibular cast to the articulator and adjust the lateral and horizontal condylar guidances.

Deprogramming of the mandible from a learned pattern of neuromuscular memory to change it from a tooth guided (ICP) to a joint guided (CRCP) movement facilitates easier maneuver into Centric closure and therefore identifying any prematurities and deflecting contacts. . Permissive occlusal splints are excellent deprogramming devices and also of diagnostic value in cases where the vertical dimension is planned to be increased. Guiding the mandible into centric closure is achieved by one of many methods like chin point guidance ${ }^{3}$, Dawson's bimanual manipulation ${ }^{4}$ Tongue to palate maneuvre ${ }^{3}$. Many recording media are available for interocclusal registration like plaster, thermoplastic waxes, zinc oxide eugenol pastes, silicones and polyethers. For its ease of manipulation and non-compressibility after setting, Aluwax is a relatively convenient material for registration. Centric records can be made with bimanual manipulation and a wax record in patients who exhibit easy movement along the retruded arc. Anterior stop techniques such as tongue spatula, modified leaf gauge ${ }^{5}$ acrylic jig ${ }^{6}$ or Jones bite frame $^{4}$ can be used in patients showing difficulty in movement along the retruded arc. Analyze the occlusion on the articulated casts, identify and remove sequentially any prematurities and interferences before the Diagnostic wax up is started after the preparation on the gypsum teeth. Wax is built up and contoured to the selected scheme of occlusion and the anterior guidance modified therein in form and position. ${ }^{7}$ Putty Silicone indices are made on the Diagnostic wax up models, one complete for fabrication of the temporaries ${ }^{8}$ and the other sectioned to aid as a guide during tooth preparation.

The Simultaneous full arch technique requires restoring both the arches at once with complete arch 
prepared dies and provides the convenience of flexibility in an occlusal plane, occlusal scheme, embrasures, crown contours and esthetics which are restricted in the Quadrant/segmental technique. However it is arduous and requires a full arch anesthesia. Canine guided occlusion is preferred in most full mouth rehabilitations because of the relative ease of fabrication and various studies ${ }^{9,10,11,12}$ support its practice. However in cases where the canines are missing or are compromised by developmental deficits such as short roots, malocclusion, marked jaw size discrepancy and periodontal disease, Group function provides for a more stable and self-maintaining scheme of occlusion and distributes the force over greater number of teeth on the mediotrusive side. Group function or Unilateral balanced occlusion has its origin in the works of Schuyler, ${ }^{13}$ Pankey, Mann, ${ }^{14}$ Ramfjord ${ }^{15}$ etc. This ensures that there is distribution of contacts on all the posterior teeth in centric position thereby applying forces along their long axes. Disocclusion occurs in the posteriors during protrusion and during lateral movements disocclusion on the non-working side occurs against the canine and buccal cusps of maxillary and mandibular premolars and molars on the working side. Group function on the working side distributes the occlusal load onto several teeth and the absence of contacts on the non-working side prevents destructive oblique forces on the teeth. However developing Group function requires greater clinical and technical precision and is relatively difficult to achieve.

\section{CONCLUSION:}

Optimum oral health should be the prime objective of all rehabilitation procedures because the ultimate goal will always be to restore the mouth to health and function and preserve this status throughout the life of the patient.

\section{REFERENCES:}

1. Teotia SP, Teotia.M. Endemic fluorosis in India : A challenging national health problem. J.Assoc Physicians India 1994;32:347-352

2. Nathan LD. Advances in functional occlusion rehabilitation. J Prosthet Dent 1956;6: 252-258

3. Stewart RK. Clinical removable Partial Prosthodontics. IEA Publishers, St. Louis, 1997.

4. Peter.E.Dawson. Evaluation, diagnosis and treatment of occlusal problems. The C.V.Mosby Company, 1989.
5. Woelfel and Lee. Suggested modification for the leaf wafer system. J Prosthet Dent 1991;65:287-291.

6. Lodge Le and Mahan PD. Study of mandibular movement from centric occlusion to maximum intercuspation. J Prosthet Dent 1967;18:19-30.

7. Irad DZ and Francis AP. Full mouth reconstruction fixedremovable. Dent Clin North Am 1987;31-35.

8. Martin D and H. Cardash. Transferring anterior occlusal guidance to the articulator.J Prosthet Dent 1983;61:282-285.

9. Lucia CV.The Gnathological concepts of articulation. Dent Clin North Am 1962;183-197.

10. D.Amico. Functional occlusion of the natural teeth of man. J Prosthet Dent 1961;2:899-901.

11. Stallard H and Stuart CE. Conceots of occlusion. Dent Clin North Am 1963;11:591-606.

12. Mann $A$, Chan $C$ and Miralles R. Influence of group function and canine guidance on EMG activity of elevator muscles. J Prosthet Dent 1987;57:494-500.

13. Schuyler $\mathrm{CH}$. Freedom in centric. Dent Clin North Am 1969;13:681-686.

14. Mann AW and Pankey LD. The PM philosophy of occlusal rehabilitation. Dent Clin North Am 1963;7:621-629.

15. Ash MM and Ramfjord SP. An introduction to functional occlusion, 1982, W.B. Saunders.

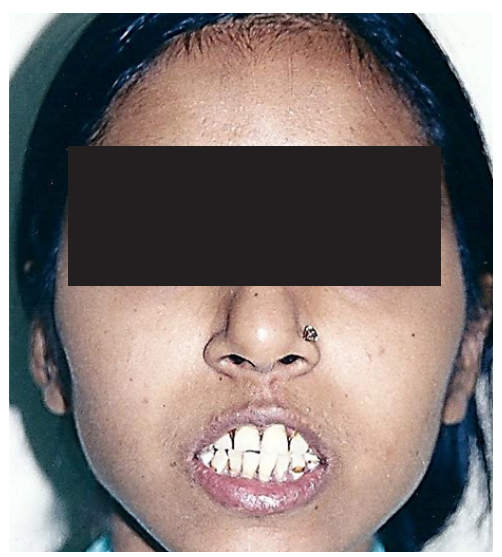

Fig 1. Pre-treatment facial view

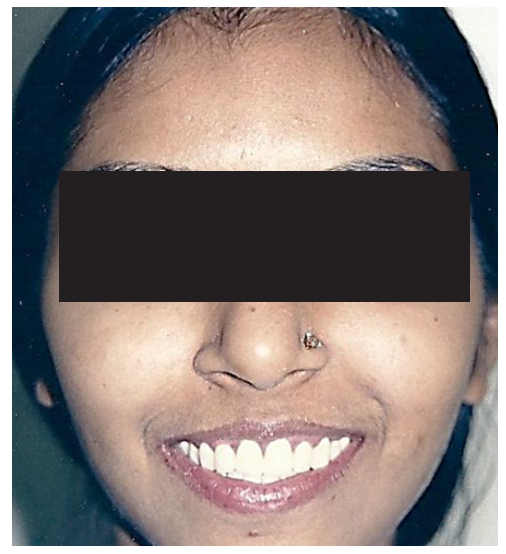

Fig 2. Post-treatment facial view 

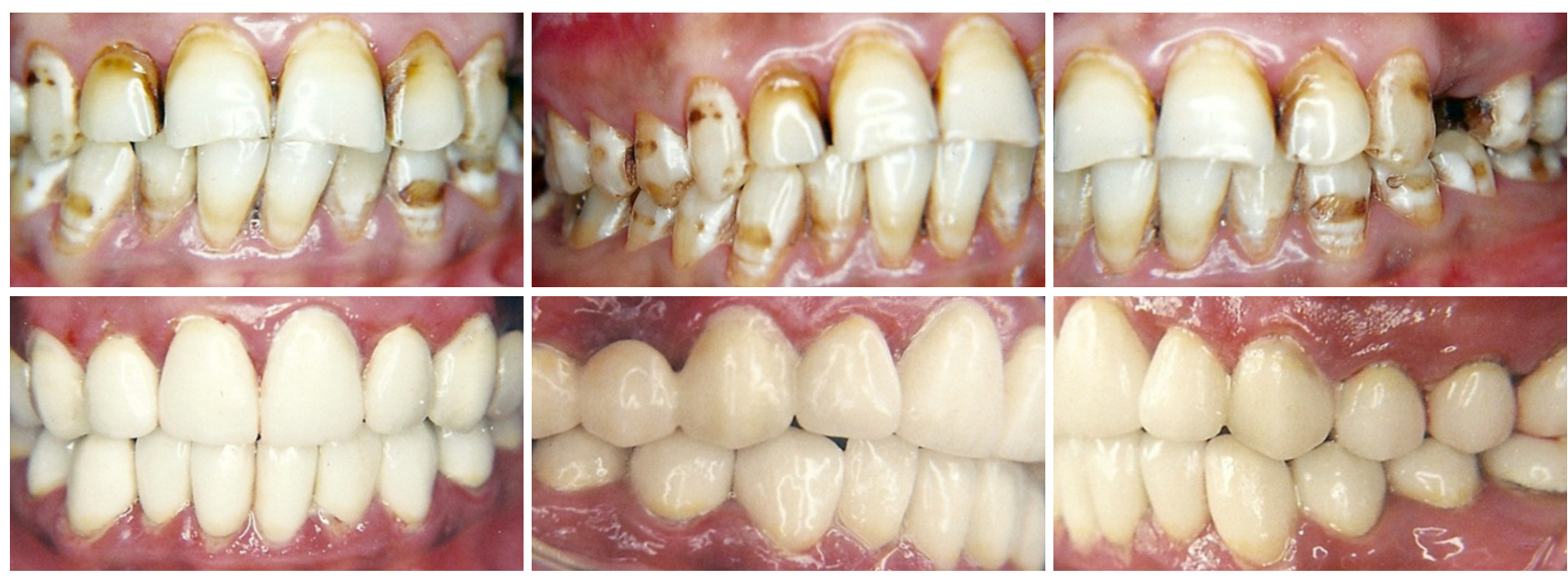

Fig 3. Pre-treatment and Post-treatment intra oral views

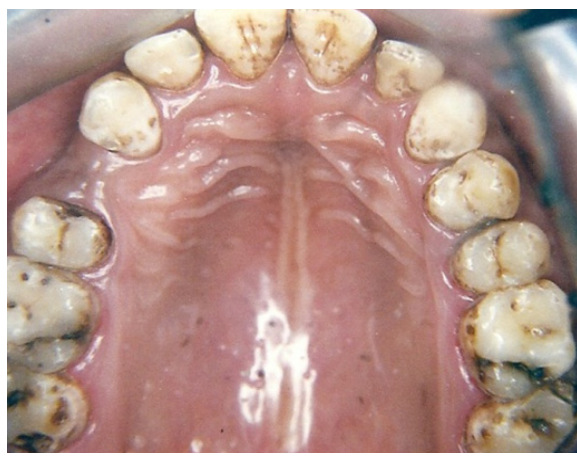

Fig. 4. Pre-treatment Maxillary occlusal view

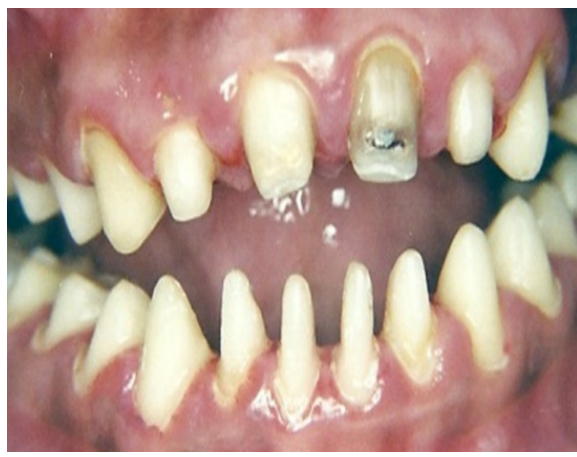

Fig.7. Simultaneous full arch preperation

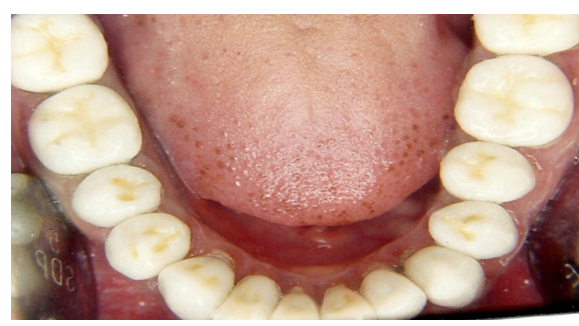

Fig.10. Post-treatment Mandibular occlusal view

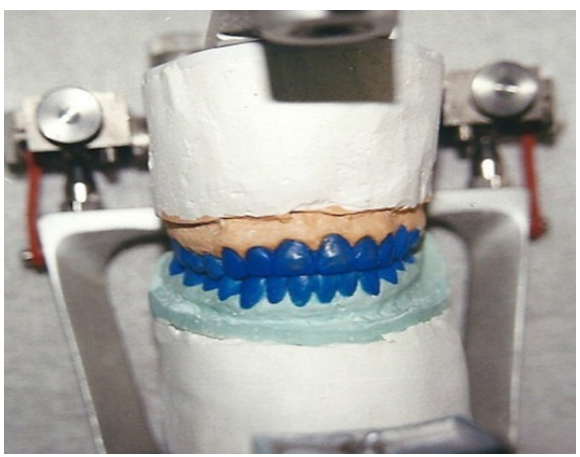

Fig. 5. Diagnostic Wax-up

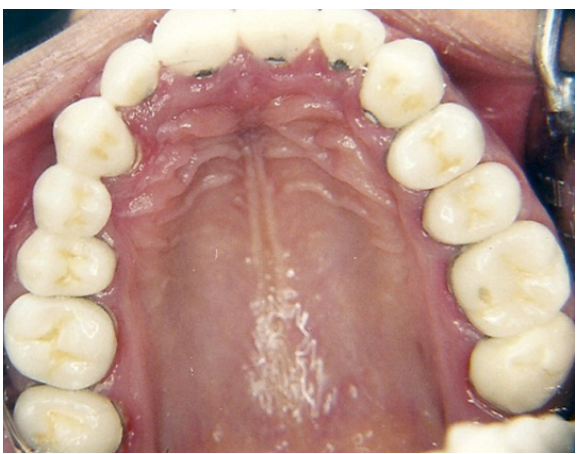

Fig.8. Post-treatment Maxillary occlusal view

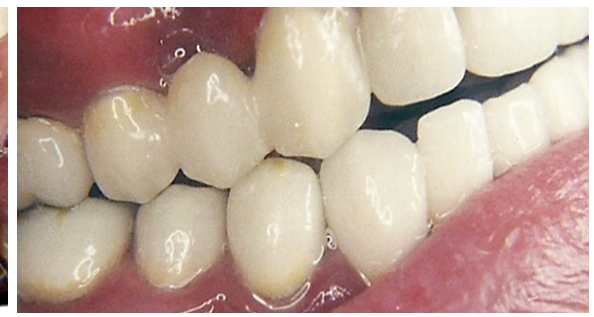

Fig.11. Working side group function contacts on the left side

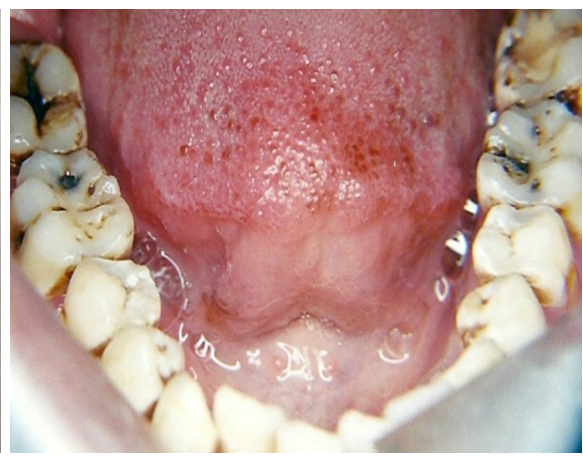

Fig. 6. Pre-treatment Mandibular occlusal view

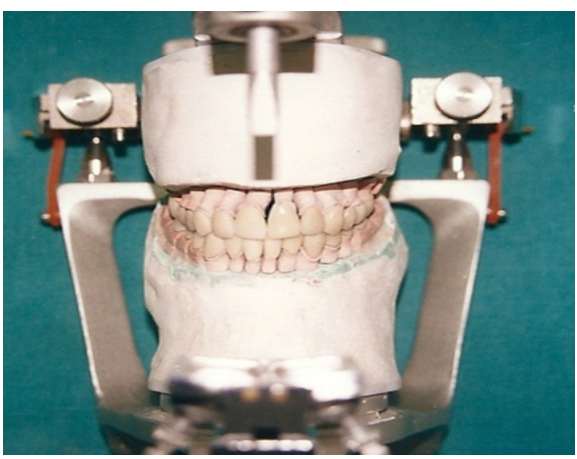

Fig. 9. Full arch dies

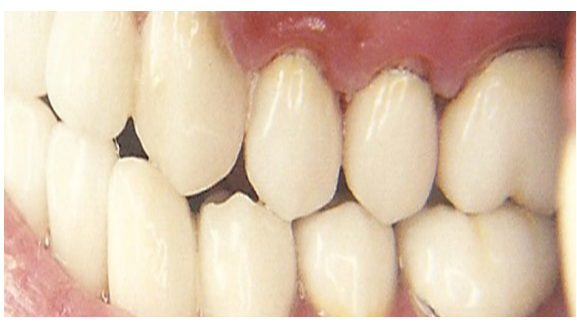

Fig.12. Working side group function contacts on the right side

IJDA, 3(3), July-September, 2011 